\title{
A Practical Approach to Detect a Fastidious but Virulent Microorganism in Infective Endocarditis of Children: Cooperation
}

The Editor,

Sir,

Abiotrophia defectiva previously classified as nutritionally variant streptococcus is a fastidious Gram-positive bacteria that was described in 1995 (1). Little is known about the pathogenesis and clinical presentation of this organism in children (2). Abiotrophia endocarditis (AE) is very rare in children without underlying heart disease (3).

A fourteen-year-old previously healthy girl was admitted to hospital with fatigue and fever for two weeks. She had good oral hygiene and no history about cardiac diseases and indwelling central catheters. On examination, she had paleness, fever $\left(38.2^{\circ} \mathrm{C}\right)$ and $3 / 6$ holosystolic murmur which was heard best in the apex. She did not have cutaneous signs. Fundii were normal. Her hemoglobin was $9.5 \mathrm{gr} / \mathrm{dl}$, total leukocyte count was 10.900/ml, erythrocyte sedimentation rate was $39 \mathrm{~mm} / \mathrm{h}$ and serum C-reactive protein was $48 \mathrm{mg} / \mathrm{L}$. Telecardiography was normal. Echocardiogram showed moderate mitral insufficiency and a large vegetation $(21 \times 16 \mathrm{~mm})$ on mitral valve (Figure).

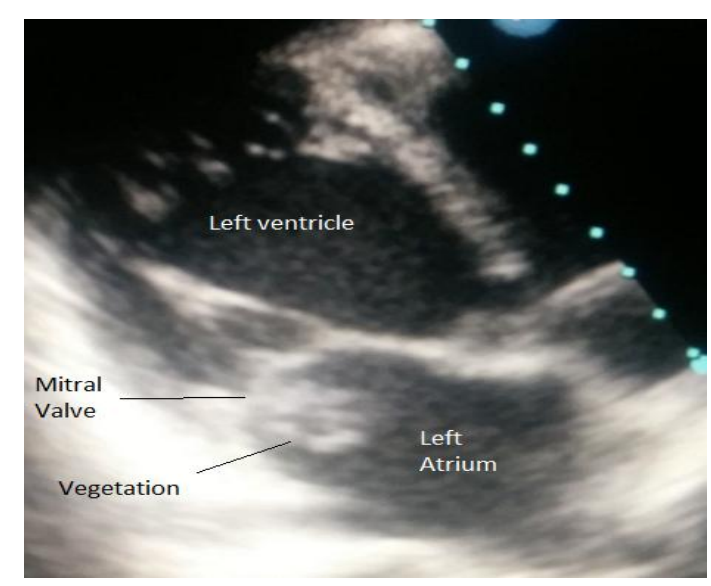

Figure: Appearence of vegetation on the atrial side of mitral valve in two-dimensional echocardiography 
A provisional diagnosis of infective endocarditis (IE) was made. Gentamycin and ceftriaxone were started as empiric antibiotherapy. Four blood cultures were drawn with adequate technique and microbiology department was informed about the patient. Patient was referred to a another center for surgical intervention. Microorganism grew on 5\% sheep blood agar plates from all blood cultures and Abiotrophia defectiva was identified with Matrix-Assisted Laser Desorption/Ionization Time-of-Flight Mass Spectrometry (MALDI-TOF MS) system. There is not a standardized susceptibility testing method for Abiotrophia and there is a poor correlation between in vitro and clinical responses $(4,5)$. The antibiotic sensitivity was tested according to streptococcus and was found to the following: cephtriaxon, clindamycin, erithromycin, linezolide, vancomycin. The results were shared with the center that patient was referred to.

Abiotrophia accounts for 5-6\% of all cases of endocarditis but this value is likely an underestimation, because Abiotrophia is a fastidious organism and might fail to grow (6). It is recommended to suspect Abiotrophia as a causative agent for IE with negative blood cultures (7). Mortality and morbidity were reported significantly higher for AE (5). In a review, rates of antibiotic failure despite sensitivity, embolization, relapse after therapy, need for surgery and death were as $41 \%, 27 \%, 17 \%, 38 \%$ and $17 \%$ in $\mathrm{AE}(8)$. According to this issues, we think that it would be better to suspect Abiotrophia in IE of children without waiting negative blood culture results and inform microbiologs about clinic diagnosis immediately.

We identified Abiotrophia with MALDI-TOF MS system but we believe that informing microbiolog contributed to detection of the microorganism in all of the four blood cultures. Cooperation between the microbiologs and the clinicians is essential in identification of this fastidious and virulant microorganism for all cases with IE suspicion. 
Keywords: Abiotrophia, children, endocarditis

Oİ Şahin ${ }^{1,}$ AH Gürsu², O Ceylan ${ }^{3}$, EB Uysal Elif Bilge ${ }^{4}$

From: ${ }^{I}$ Division of Pediatric Cardiology, Department of Pediatrics, Faculty of Medicine, Cumhuriyet University, Sivas, Turkey ${ }^{2}$ Department of Pediatric Cardiology, Sivas State Hospital, Turkey ${ }^{3}$ Department of Pediatric Infectious Diseases, Sivas State Hospital, Turkey

${ }^{4}$ Department of Medical Microbiology, Faculty of Medicine, Cumhuriyet University, Sivas, Turkey

Correspondence: Dr IO Şahin, Division of Paediatric Cardiology, Department of Paediatrics, Faculty of Medicine, Cumhuriyet University, Sivas, Turkey Fax: +90442 23609 68,e-mail: rfnshn@yahoo.com

\section{REFERENCES}

1. Kawamura Y, X Hou, F Sultana, S Liu, H Yamamoto, T Ezaki. Transfer of Streptococcus adjacens and Streptococcus defectivus to Abiotrophia gen. nov. as Abiotrophia adiacens comb. nov. and Abiotrophia defectiva comb. nov., respectively. Int J Syst Bacteriol 1995; 45: 798-803.

2. Michelow IC, McCracken GH Jr, Luckett PM, Krisher K. Abiotrophia spp. brain abscess in a child with Down's syndrome. Pediatr Infect Dis J 2000; 19: 760-3.

3. Bhat DP, Nagaraju L, Asmar BI, Aggarwal S. Abiotrophia endocarditis in children with no underlying heart disease: a rare but a virulent organism. Congenit Heart Dis 2014; 9: 116-20.

4. Clark RB, Gordon RE, Bottone EJ, Reitano M. Morphological aberrations of nutritionally deficient streptococci: association with pyridoxal (vitamin $\mathrm{B}_{6}$ ) concentration and potential role in antibiotic resistance. Infect Immun 1983; 42: 414-7

5. Chang HH, Lu CY, Hsueh PR, Wu MH, Wang JK, Huang LM. Endocarditis caused by Abiotrophia defectiva in children. Pediatr Infect Dis J 2002; 21: 697-700.

6. Raff GW, Gray BM, Torres A Jr, Hasselman TE. Aortitis in a child with Abiotrophia defectiva endocarditis. Pediatr Infect Dis J 2004; 23:574-6. 
7. Ray M, Subramanian C, Ray P, Singhi P. Infective endocarditis in a child due to Abiotrophia defectivus. Indian Pediatr 2002; 39: 388-92.

8. Stein DS, Nelson KE. Endocarditis due to nutritionally deficient streptococci: therapeutic dilemma. Rev Infect Dis 1987; 9: 908-16. 